Proceedings of the 5th Workshop on Quantum Chaos and Localisation Phenomena, Warsaw, Poland, May 20-22, 2011

\title{
Chaos in the Brain
}

\author{
J. KŘÍǏZ \\ University of Hradec Králové, Faculty of Science, Rokitanského 62, 50003 Hradec Králové, Czech Republic
}

We describe several links between EEG data processing and quantum mechanics. Then we show examples of exploitation of methods commonly used in quantum chaos for EEG data analysis.

PACS: 02.50.Sk, 02.70.Rr, 05.45.Tp, 87.19.ie

\section{What has quantum mechanics (chaos) in common with human brain?}

The electroencephalogram (EEG) is a noninvasive medical test measuring the electrical activity of the human brain via electrodes applied to the subject's scalp. Although being used as a clinical diagnostic tool for nearly 80 years (see [1]), it is still mostly the subject of subjective visual analysis by physicians. The strict and objective analysis of EEG remains still the challenge not only for biomedical signal processing engineers, but also for mathematicians, statisticians, and physicists. Reminding the famous quote of Richard Feynman: "I think I can safely say that nobody understands quantum mechanics", we arrive at the first clear link between quantum mechanics and human brains, namely the EEG data analysis. We are dealing with the two topics that nobody completely understands.

EEG recordings represent multivariate time series, number of channels corresponds to the number of EEG electrodes. Each channel - EEG signal at one electrode (i.e. the potential difference between the electrode and some reference electrode) is a result of the synchronous electrical activity of huge number of cortical neurons. Hence, the superposition of electric waves is measured. And the superposition principle is one of the cornerstones of the quantum mechanics! The activity of cortical neurons is a result of countless discharge cascades across the network of neurons located in deeper areas of the brain. Due to this complex origin, one could expect the EEG signal to be the approximately superposition of random waves. It was really demonstrated that the structures emerging in the brain's visual cortex of animals can be described by random Gaussian fields, see [2,3]. The same random waves are known from quantum chaotic systems (this was first conjectured by Berry [4]), such are e.g. quantum billiards, see e.g. [5].

EEG signal, such as most real world signals, is non-stationary in the sense that it has highly complex time-varying characteristics. The monitoring of its frequency variation with time is of immense importance. To ob-

* e-mail: jan.kriz@uhk.cz tain the frequency variation of non-stationary signal, one should segment the signal into quasi-stationary components and apply standard Fourier transform to them. The longer the segment is, the better accuracy in frequency we obtain. This fact that a function and its Fourier transform cannot both be highly concentrated is known as an "uncertainty principle". In the (EEG) signal processing, the uncertainty principle establishes limits to which the "instantaneous frequency" of a signal can be determined, see [6] and the references therein. The quantum mechanical parallel is of course famous Heisenberg uncertainty principle - the fact that the particle's position and momentum cannot be measured simultaneously.

Just mentioned similarities suggest that the exploitation of mathematical tools commonly used in quantum mechanics (quantum chaos) for EEG signal processing can be auspicious. In this paper we briefly review the application of the random matrix theory (RMT) to the EEG signal analysis (Sect. 2) and then present a maximum-likelihood based algorithm for estimating the evoked potential response from a multiepoch EEG data (Sect. 3).

\section{Random matrix theory and EEG}

RMT originated in nuclear physics and was conceived as a statistical approach to systems with many degrees of freedom. Nowadays RMT has applications in many disciplines of science. However, one of the most important discoveries in RMT was its connection to quantum chaos - RMT describes the spectral statistics of the systems whose classical counterparts exhibit chaotic behavior, for more details see the review paper [7].

According to our knowledge, RMT was used to analyze the EEG signal for the first time in the paper [8] by Šeba. In particular, statistical properties of spectra of EEG cross-channel correlations matrices were compared with the predictions of RMT. Here we review the results of this paper.

The analyzed objects were the correlation matrices $C_{l, m}(T)$ of the EEG signal,

$$
C_{l, m}(T)=\sum_{j=N_{1}}^{N_{2}} x_{l}\left(t_{j}\right) x_{m}\left(t_{j}\right),
$$


where $x_{l}\left(t_{j}\right)$ denotes the EEG signal measured at the channel $l$ at time $t_{j}$ and the sum runs over $j=N_{1}, N_{1}+$ $1, \ldots N_{2}$ for which $t_{j} \in(T, T+\Delta)$. The length $\Delta$ of the time interval used to evaluate the correlation matrix is related to the rate with which the brain works and was set to $150 \mathrm{~ms}$. Data from clinical 19-channel EEG device were analyzed, each measurement lasted for 15-20 min. This time interval was divided into approximately 7000 not overlapping stationary windows over which the correlation matrix was evaluated. In such a way the ensembles of 7000 correlation matrices for each measurement were obtained. Due to the individuality of the EEG signal and also due to the problems related to the technique the electrodes are attached to the scalp one cannot mix together data from different measurement sessions. This means that for different measurements, different matrix ensembles were obtained. The data from 90 different measurements were inspected, some of them were influenced by the visual stimulation.

The simplest property of the eigenvalues family related to a matrix ensemble is the eigenvalue density, which is known to be dependent on the underlying data. Since EEG is not a random signal, but on the contrary, it is synchronized and correlated by the corresponding brain activity, one might expect that the spectral density will not exhibit generic and subject-independent features. Surprisingly, this is not true at all. For small eigenvalues the spectral density indeed depends on the measured subject. It displays nevertheless at all subjects a profound and subject-independent algebraic tail for large eigenvalues. This is a surprise since one would expect just the opposite behavior. Specifically, the small eigenvalues of the correlation matrix feel the influence of the system noise, the spectral density should therefore display universal behavior for them. The large eigenvalues on the other hand contain the information about significant correlations and hence about processes in the brain. Consequently, one would expect a nonuniversal and subject-dependent behavior. The universal behavior of the spectral density for large eigenvalues means that the seemingly individual brain activity contains some common level of synchronization.

Furthermore, Šba focused on two statistical distributions widely used in the field of quantum chaos - level spacing and number variance distributions. These distributions obtained from the ensembles of EEG correlation matrices were compared with the well-known and universal distributions for Gaussian orthogonal ensemble (GOE). As we have already mentioned the EEG signal is subject dependent. Nevertheless, the spectral statistical properties of the correlation matrices are universal. In particular, the behavior of the level spacing distribution and number variance obtained from the EEG data of healthy subjects without the visual stimulation are in very good agreement with the predictions of GOE. The visual stimulation is expected to change the correlation pattern of the data. Nevertheless, it turned out that these changes are too subtle to influence the level spac- ing distribution. A very good agreement with the RMT in all subjects was reported. The number variance is, however, quite sensitive and changes when the subject is visually stimulated, the random matrix prediction is in this case valid for nonstimulated data only.

This result is of interest since the number variance may change not only due to an external stimulation but also when the correlation ensemble is influenced, for instance, by some pathological process.

\section{Maximum likelihood estimation and evoked response EEG potentials}

Maximum likelihood estimation (MLE) is a standard statistical method dating back to 1920's. It is a versatile tool covering wide range of applications, but its benefits are apparent particularly in the quantum physics. For example, in the quantum state reconstruction, for a given set of measurements, the most likely quantum state is estimated. As for the quantum chaos branch, the MLE was used e.g. for the implementation of the quantum state reconstruction to the quantum kicked top (see [9]) or to study chaotic scattering in overlapping resonators (see [10]).

The brain response to an external stimulus measured by EEG is called evoked response (or event related) potential (ERP). When an external stimulus is presented to a measured subject, several parts of the brain that are involved in processing the stimulus show increased neuronal activity and thus act as the generators of the brain response. The signal has typically extremely low signal-to-noise ratio (SNR), the signal of interest (response of the brain to the stimulus) is embedded within the ongoing background EEG. The visual evoked potential (VEP), a subset of ERPs, has become a commonly used tool in both research and clinical studies regarding the functional integrity of visual system, see e.g. [11, 12].

When the same stimulus is applied repeatedly, the brain response is typically estimated from the recorded data by averaging technique [13]. The underlying assumption behind averaging is that the signal of interest is the same across trials, time locked to the stimulus, while the noise is independent. However, it is known that the constant response assumption is false, i.e. identical stimuli do not necessarily evoke identical responses. In general, ERP waveform, amplitude and latency can vary appreciably in time [14].

In ERP analysis, the on-going EEG is usually treated as background activity or noise. Although the noise can be regarded independent from trial to trial, it is highly (both spatially and temporally) correlated, see [15-17] and references therein. Moreover, it has been shown that the noise is not entirely uncorrelated with event-related activity [18]. Thus, appropriate space-time processing should exploit the noise coloration.

Numerous papers dealing with the application of maximum likelihood (ML) technique to ERPs have been published during last decade. In [19], an ML model is formu- 
lated yielding estimators of amplitude and latency variations of single trials in a multiepoch ERP experiment. Restrictions of the paper are, however, that it is based on an assumption of uncorrelated background noise and the response is estimated separately for each channel (electrode). It means that no spatial structure is involved in the model. In reality, at least the signals measured on nearby electrodes must be strongly correlated, since the signals have partially the same sources.

In the paper [16], De Munck et al. derived an ML estimator for the amplitude jitters on the assumption that the background noise is both spatially and temporally correlated. However, they did not incorporate the latency variations to their model. And again, the model is spatially local, the estimates of particular channels are obtained independently. The spatial structure is contained only in the spatial covariance matrix.

The ML approach exploiting spatial correlations of the signal was presented by Baryshnikov et al. in the paper [15]. They model the brain response to a stimuli as a low rank or structured mean. The rank of the response is assumed to be much smaller than the number of spatial channels (electrodes), but with unknown covariance. On the other hand, the main disability of their model is the above mentioned false constant response.

Extending the method of Baryshnikov et al. we present a ML approach for estimating the brain response to external stimuli modeled by a low rank mean, in which amplitude and latency variability is also allowed. We exploit the procedure described in [15], however we add to it two more steps for amplitude and latency estimating.

\subsection{The Baryshnikov et al. model}

In this section we briefly describe the model of Baryshnikov et al. For more detailed description we refer to their paper [15] and references therein.

We consider $J$ observations of response to the same stimulus (epochs). The signal of interest is considered to be repeatable component of each epoch. Denoting the number of spatial channels by $N$ and number of time samples per epoch by $T$, the data for $j$-th epoch is represented by the $N \times T$ matrix $\boldsymbol{X}_{j}=\boldsymbol{S}+\boldsymbol{W}_{j}$, where $\boldsymbol{S}$ is the matrix of signal samples and $\boldsymbol{W}_{j}$ is the matrix of noise samples in the $j$-th epoch.

It is assumed that signal time series in each channel (rows of the matrix $\boldsymbol{S}$ ) lies in the space spanned by the columns of the known $T \times L$ matrix $\boldsymbol{C}$. The set of temporal basis vectors (columns of $\boldsymbol{C}$ ) is constructed using the frequency band of interest.

Similarly, let the columns of unknown $N \times P$ matrix $\boldsymbol{H}$ be a basis for the $P$-dimensional space in which the columns of the signal matrix $\boldsymbol{S}$ lie. The structure of $\boldsymbol{H}$ is not anyhow restricted. The free parameter $P$ (rank of the matrix $\boldsymbol{H}$ ) is chosen empirically.

Thus, the signal matrix can be expressed in the form

$$
\boldsymbol{S}=\boldsymbol{H} \Theta \boldsymbol{C}^{\mathrm{T}},
$$

where $\Theta$ is a $P \times L$ matrix of unknown signal amplitude parameters. Let $\boldsymbol{X}=\left[\boldsymbol{X}_{1} \boldsymbol{X}_{2} \ldots \boldsymbol{X}_{J}\right]$ be the $N \times J T$ full data matrix formed from all epochs. Since the signal of interest is assumed to be identical in each epoch, it is possible to expand the signal component of the full dataset in terms of low-rank model $[S \boldsymbol{S} \ldots \boldsymbol{S}]=\boldsymbol{H} \Theta \boldsymbol{D}^{\mathrm{T}}$, where

$$
\boldsymbol{D}^{\mathrm{T}}=\left[\boldsymbol{C}^{\mathrm{T}} \boldsymbol{C}^{\mathrm{T}} \ldots \boldsymbol{C}^{\mathrm{T}}\right]
$$

is the $L \times J T$ matrix.

The noise matrices $\boldsymbol{W}_{j}$ are assumed to be normally distributed, zero mean, independent of one another, with unknown spatial covariance matrix $\boldsymbol{R}_{N}$ and prior the estimation of $\boldsymbol{S}$ known (or estimated) temporal covariance matrix $\boldsymbol{R}_{\mathrm{T}}$. In the following we will assume $\boldsymbol{R}_{\mathrm{T}}=\boldsymbol{I}$, however the whole procedure is applicable to the case $\boldsymbol{R}_{\mathrm{T}} \neq \boldsymbol{I}$ by transforming $\boldsymbol{X}_{j}$ to $\boldsymbol{X}_{j} \boldsymbol{R}_{N}^{-1 / 2}$. The total noise covariance matrix is modeled as the Kronecker product of spatial and temporal matrices. Hence the probability density function for the data matrix $\boldsymbol{X}$ may be written

$$
\begin{aligned}
& f\left(\boldsymbol{X} ; \boldsymbol{R}_{N}, \boldsymbol{H}, \boldsymbol{\Theta}\right)=\frac{1}{(2 \pi)^{\frac{N T J}{2}} \operatorname{det}\left(\boldsymbol{R}_{N}\right)^{\frac{T J}{3}}} \\
& \quad \times \exp \left(-\frac{1}{2} \operatorname{Tr}\left(\boldsymbol{R}_{N}^{-1}\left(\boldsymbol{X}-\boldsymbol{H} \boldsymbol{\Theta} \boldsymbol{D}^{\mathrm{T}}\right)\right.\right. \\
& \left.\left.\quad \times\left(\boldsymbol{X}-\boldsymbol{H} \boldsymbol{\Theta} \boldsymbol{D}^{\mathrm{T}}\right)^{\mathrm{T}}\right)\right) .
\end{aligned}
$$

The ML estimate of $\boldsymbol{H}, \boldsymbol{\Theta}$ and $\boldsymbol{R}_{N}$ are obtained by maximizing (3) for the given data vector $\boldsymbol{X}$. We skip the derivation here, however, the estimates of all mentioned matrices and thus also of the signal matrix $\boldsymbol{S}$ are obtained analytically.

\subsection{Our model}

We start from the low rank Baryshnikov et al. model. However we incorporate the amplitude and latency variations to it. Thus the varied signals of interest are modeled in the form

$$
\boldsymbol{S}_{j}=k_{j} \boldsymbol{S} \boldsymbol{L}^{\alpha_{j}}=k_{j} \boldsymbol{H} \boldsymbol{\Theta} \boldsymbol{C}^{\mathrm{T}} \boldsymbol{L}^{\alpha_{j}},
$$

where $\boldsymbol{H}, \boldsymbol{\Theta}, \boldsymbol{C}$ have the same meaning as in (1), $k_{j}$ is a real positive epoch dependent amplitude weight, $\alpha_{j}$ is an integer latency lag and the $T \times T$ matrix $\boldsymbol{L}$ is recurrent shift matrix

$$
\boldsymbol{L}=\left(\begin{array}{ccccc}
0 & 1 & 0 & \ldots & 0 \\
0 & 0 & 1 & \ldots & 0 \\
\vdots & \vdots & \vdots & \ddots & \vdots \\
0 & 0 & 0 & \ldots & 1 \\
1 & 0 & 0 & \ldots & 0
\end{array}\right)
$$

The factors $k_{j}$ obviously describe the variation of the response amplitudes, see e.g. [16, 19]. It is easy to check that the right multiplication of a matrix by $\boldsymbol{L}^{\alpha_{j}}$ causes the (cyclic) shift of columns of the matrix by $\alpha_{j}$ to the right. Since each row of the matrix $\boldsymbol{H} \Theta C^{\mathrm{T}}$ is a time series representing the time evolution of the response, its 
shift to the right by $\alpha_{j}$ means the "delay" of the response by $\alpha_{j} / f$, where $f$ is a sampling frequency of the recordings. (In our experiment, we have used the device with the sampling frequency $1 \mathrm{kHz}$, thus the value of $\alpha_{j}$ is numerically equaled to the latency lag expressed in $\mathrm{ms}$.) The recurrent shift operator (not just shift operator) is used, because it is easier to handle it. In particular, it is invertible, thus we have just one operator for the latency lag in both positive and negative directions (with positive, respectively negative values of $\alpha_{j}$ ). On the other hand, the recurrent shift operator copies the last column of the matrix before the shift to the first column. Therefore we have to assume that the latency lags $\alpha_{j}$ are small in comparison with the number of time samples per epoch $T$ and the signal of interest is zero at the beginning and end of each epoch. However, these assumptions are naturally satisfied. Usually ERP data contains in each epoch some signal free (prestimulus) samples and the length of the record of each epoch is longer than expected period of the brain response.

The amplitude and latency variations can be easily incorporated to the matrix $\boldsymbol{D}$. Instead of (2) we have thus

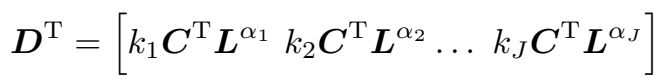

and the probability density function remains formally the same as (3). However, now it has more parameters to be estimated by MLE method, namely $J$ amplitude weights $k_{j}$ and $J$ latency lags $\alpha_{j}$.

Since we have formally the same probability density function as Baryshnikov et al. we can use their procedure to estimate $\boldsymbol{H}, \boldsymbol{\Theta}$ and $\boldsymbol{R}_{N}$. The ML estimators for $k_{j}$ and $\alpha_{j}(j=1, \ldots, J)$ are found by setting the corresponding derivatives of (3) equal to zero and solving the estimators from resulting equations. Doing so for $k_{j}$ we have after straightforward calculation

$$
\begin{aligned}
& k_{j}= \\
& \frac{\operatorname{Tr}\left(\boldsymbol{R}_{N}^{-1}\left(\boldsymbol{X}_{j}\left(\boldsymbol{L}^{\alpha_{j}}\right)^{\mathrm{T}} \boldsymbol{C} \boldsymbol{\Theta}^{\mathrm{T}} \boldsymbol{H}^{\mathrm{T}}+\boldsymbol{H} \boldsymbol{\Theta} \boldsymbol{C}^{\mathrm{T}} \boldsymbol{L}^{\alpha_{j}} \boldsymbol{X}_{j}^{\mathrm{T}}\right)\right)}{2 \operatorname{Tr}\left(\boldsymbol{R}_{N}^{-1}\left(\boldsymbol{H} \boldsymbol{\Theta} \boldsymbol{C}^{\mathrm{T}} \boldsymbol{C} \boldsymbol{\Theta}^{\mathrm{T}} \boldsymbol{H}^{\mathrm{T}}\right)\right)} .
\end{aligned}
$$

One can easily check moreover that this value of $k_{j}$ corresponds to local maximum. Namely, the argument of the exponential function is a quadratic function of each $k_{j}$ with the negative quadratic term. For the latency lags the following equation can be derived:

$$
\begin{aligned}
& \operatorname{Tr}\left(\boldsymbol { R } _ { N } ^ { - 1 } \left(\boldsymbol{X}_{j}\left(\frac{\mathrm{d} \boldsymbol{L}^{\alpha_{j}}}{\mathrm{~d} \alpha_{j}}\right)^{\mathrm{T}} \boldsymbol{C} \boldsymbol{\Theta}^{\mathrm{T}} \boldsymbol{H}^{\mathrm{T}}\right.\right. \\
& \left.\left.+\boldsymbol{H} \boldsymbol{\Theta} \boldsymbol{C}^{\mathrm{T}} \frac{\mathrm{d} \boldsymbol{L}^{\alpha_{j}}}{\mathrm{~d} \alpha_{j}} \boldsymbol{X}_{j}^{\mathrm{T}}\right)\right)=0 .
\end{aligned}
$$

Since we are looking for just integer values of $\alpha_{j}$ we do not solve Eq. (8) and instead of it we solve the following minimization problem:

$$
\min _{\alpha_{j} \in \mathbb{Z}} \mid \operatorname{Tr}\left(\boldsymbol { R } _ { N } ^ { - 1 } \left(\boldsymbol{X}_{j}\left(\boldsymbol{L}^{\left(\alpha_{j}+1\right)}-\boldsymbol{L}^{\alpha_{j}}\right)^{\mathrm{T}} \boldsymbol{C} \boldsymbol{\Theta}^{\mathrm{T}} \boldsymbol{H}^{\mathrm{T}}\right.\right.
$$

$$
\left.\left.+\boldsymbol{H} \boldsymbol{\Theta} \boldsymbol{C}^{\mathrm{T}}\left(\boldsymbol{L}^{\left(\alpha_{j}+1\right)}-\boldsymbol{L}^{\alpha_{j}}\right) \boldsymbol{X}_{j}^{\mathrm{T}}\right)\right) \mid
$$

So we have replaced the derivative by the difference operator and we are looking for such integer value of $\alpha_{j}$, for which the left-hand side of $(8)$ is closest to zero.

Note that in (7)-(9), the symbols $k_{j}$ and $\alpha_{j}$ have been used to indicate the estimators of true values. Problems (7) and (9) have to be solved iteratively. First, $k_{j}=1$ and $\alpha_{j}=0$ for $j=1, \ldots J$ are taken. Then the first estimates of $\boldsymbol{H}, \boldsymbol{\Theta}$ and $\boldsymbol{R}_{N}$ are calculated by Baryshnikov et al. procedure. These first estimates are then substituted into (9), which is solved numerically for every $j=1, \ldots J$. Let us note that problem (9) does not depend on $k_{j}$. All mentioned first estimates are finally substituted into (7) to obtain the first estimates of all $k_{j}$. Estimates of $k_{j}$ and $\alpha_{j}$ are then substituted into (6) and the updates of $\boldsymbol{H}, \boldsymbol{\Theta}$ and $\boldsymbol{R}_{N}$ are calculated again by Baryshnikov et al. procedure, etc. In practice, it appears that a few (usually 9-16) of these iterations are sufficient to obtain a stable solution.

We have investigated the data of 5 volunteers measured by standard clinical 63-channel EEG device placed inside a Faraday trap to avoid external influence. We used the common pattern reversal stimulus according to the standard [20]. For each subject, 100 epochs lasting $666 \mathrm{~ms}$ (200 ms before the stimulus onset and 466 after it) were collected with a sampling rate of $1 \mathrm{kHz}$.

The algorithm presented in Sect. 3.2 converged in 9-16 iterations. We choose $\boldsymbol{C}$ to be a basis for signals bandlimited on $1-20 \mathrm{~Hz}$, with $L=40$. The rank of the estimator was chosen $P=5$. A quite strict stopping rule was used, implying the relative changes were smaller than $10^{-8}$. Typically this resulted in a computation time about $20 \mathrm{~min}$ (processor i920, 8 GB RAM).

Figure 1 shows a typical example of the amplitude trial-to-trial variations. The amplitude weights $k_{j}$ are plotted as a function of the number of stimulus $j$. To extract systematic behavior from all data sets, a straight line was fit to the amplitude data. The quality of these line fits were in all cases comparable to the example presented in Fig. 1. In all the cases, the slope of the line fit was negative, showing that the response are weaker at the end of the measurement session than at the beginning (so-called habituation). This result is in accordance with [16].

The typical example (the same subject as in Fig. 1) of the latency trial-to-trial variations is depicted in Fig. 2. The latency lags $\alpha_{j}$ are plotted as a function of the number of stimulus $j$. Again, a straight line was fit to extract systematic behavior from all data sets and its behavior is reproducible across all measured objects. In all the cases, the slope of the line fit was positive, showing that the response latency has an increasing trend from the beginning to the end of the measurement session. This observation is in accordance with [19]. The histogram of latency lags shows that its right-tail is longer than the left one. 

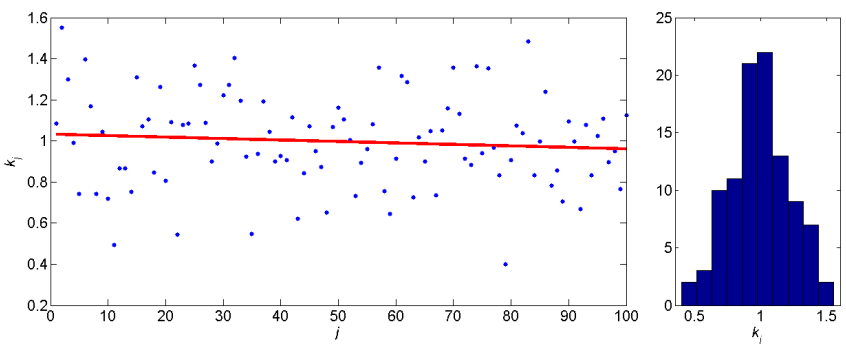

Fig. 1. Trial dependence of amplitude weights is shown for typical example together with its histogram. The vertical scale is such that 1 implies the traditional constant response model. On the horizontal axis there is a number of epoch. The straight red line is the least squares fit of the data.
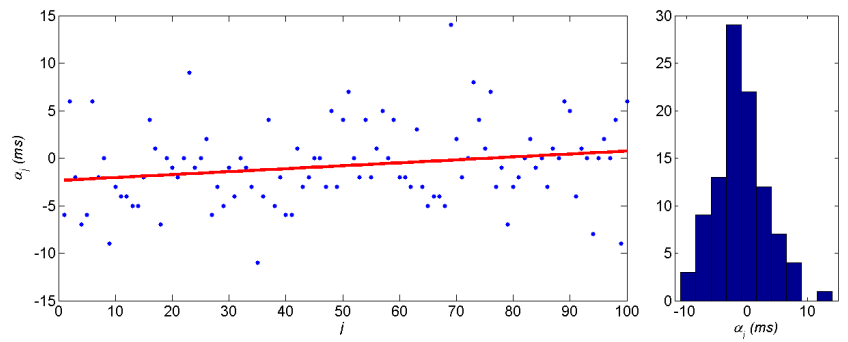

Fig. 2. Trial dependence of latency lags is shown for typical example together with its histogram. The vertical scale is such that $0 \mathrm{~ms}$ implies the traditional constant response model. On the horizontal axis there is a number of epoch. The straight red line is the least squares fit of the data.

The applications of our model to real pattern reversal VEP data show a systematic weakening of the signal amplitudes. This negative trend in response can be explained as habituation or nerve fatigue effects as was reported e.g. in [21]. In the same time the habituation causes the increase of response latencies. Again, this effect was described in literature [21]. However, most of studies of habituation contain several implicit assumptions that are avoided with the ML model presented here. The price to be paid is the relative long computation time.

We believe that our method can be useful in studying the ERPs when the subject is instructed to perform some task depending on the stimulus. In fact, ERPs consist of exogenous and endogenous components, see [17] and references therein. Exogenous components are obligatory responses which result on the presentation of physical stimuli. The endogenous components manifest the processing activities which depend on the stimuli's role within the task being performed by subject. The latter exhibit more pronounced trial-to-trial variations.

\section{Acknowledgments}

This work was supported by the Czech Science Foundation grant 202/06/P130, by the project LC06002 of the Ministry of Education, Youth and Sports of the Czech Republic and the project of the specific research Faculty of Education, University of Hradec Králové. The author is very grateful to Petr Šeba for the fruitful discussions and suggestions. The help of Jan Kremláček is also gratefully acknowledged.

\section{References}

[1] B.E. Swartz, E.S. Goldensohn, Electroenceph. Clin. Neurophysiol. 106, 173 (1998).

[2] F. Wolf, T. Geisel, Nature 395, 73 (1998).

[3] M. Schnabel, M. Kaschube, S. Lowel, F. Wolf, Eur. Phys. J. Special Topics 145, 137 (2007).

[4] M.V. Berry, J. Phys. A 10, 2083 (1977).

[5] P. Šeba, Phys. Rev. Lett. 64, 1855 (1990).

[6] D.L. Donoho, P.B. Stark, SIAM J. Appl. Math. 49, 906 (1989).

[7] T. Guhr, A. Müller-Groeling, H.A. Weidenmueller, Phys. Rep. 299, 189 (1998).

[8] P. Šeba, Phys. Rev. Lett. 91, 198104 (2003).

[9] S.T. Merkel, C.A. Riofrí, S.T. Flammia, I.H. Deutsch, Phys. Rev. A 81, 032126 (2010).

[10] B. Dietz, T. Friedrich, H.L. Harney, M. Misky-Oglu, A. Richter, F. Schäfer, H.A. Weidenmüller, Phys. Rev. E 78, 055204 (2008).

[11] W.A. Cobb, G.D. Dawson, J. Physiol. 152, 108 (1960).

[12] H.G. Vaughan, R.C. Hull, Nature 206, 720 (1965).

[13] J.I. Aunon, C.D. McGillem, D.G. Childers, CRC Crit. Rev. Bioeng. 5, 323 (1981).

[14] D.H. Lange, H.T. Siegelmann, H. Pratt, G.F. Inbar, IEEE Trans. Biomed. Eng. 47, 822 (2000).

[15] B.V. Baryshnikov, B.D. Van Veen, R.T. Wakai, IEEE Trans. Biomed. Eng. 51, 1981 (2004).

[16] J.C. de Munck, F. Bijma, P. Gaura, C.A. Sieluzycki, M.I. Branco, R.M. Heethaar, IEEE Trans. Biomed. Eng. 51, 2123 (2004).

[17] C.N. Gupta, R. Palaniappan, Computat. Intel. Neurosci. 2007, 28692 (2007).

[18] M. Kutas, G. McCarthy, E. Donchin, Science 197, 792 (1977).

[19] P. Jaškowski, R. Verleger, IEEE Trans. Biomed. Eng. 46, 987 (1999).

[20] J.V. Odom, M. Bach, C. Barber, M. Brigell, M.F. Marmor, A.P. Tormene, G.E. Holder, Veagan, Docum. Ophthalm. 108, 115 (2004).

[21] H. Orbig, H. Israel, M. Kohl-Bareis, K. Uludag, R. Wenzel, B. Müller, G. Arnold, A. Villringer, NeuroImage $\mathbf{1 7}, 1$ (2002). 